\title{
Is tendency to recurrently abort, a rejected malignant activity in developing embryonic tissue?
}

\author{
Kishore Goswami* \\ Bhopal University, MP, India
}

\begin{abstract}
This short paper reemphasizes the importance of certain chromatin dots (named as Marker dots) which are seen emanating from chromosomes in lymphocyte cultures of various syndromes and persons showing early signs of malignancy as well as among a greater majority of women who exhibited recurrent abortions (03 or more abortions). Those couples who were detected to show history of recurrent abortions but were otherwise fertile and normal (from reproductive points of view) were referred to our genetic clinic by gynecologists and were investigated for chromosome scrutiny. Assuming that both husband and wife constitute one unit (equally responsible: genetically) for the result of early termination of pregnancy, we had carried out simultaneous study on chromosomes by lymphocyte cultures. During investigations we had put a questionnaire asking separately that if any one of them had a history of any type of cancer in their family. Out of 281 couples investigated 207 couples were positive with cancer history and 72 were without any cancer history. Also, there were 35 couples who showed abortions but without cancer history. The difference is highly significant (Chi square $/ \chi^{2}=6.635$ at $1 \mathrm{df} ; \mathrm{p}<0.01$ ). That cancer history in the family and recurrent abortions are intricately related becomes more vivid and well established by observations that out of 281 couples 193 couples are those who exhibit 03 or more abortions and among them, only 18 couples show no cancer history in the family. Precisely, 175 couples have cancer-history while only 18 were without it, indicating that cancer history in the family increases tenfold possibility of recurrent abortions. Furthermore, earlier studies had reported marker dots to be positively associated with women showing 03 to 07 abortions and have been referred to be indicators of chromosomal mutagenesis; because these have been found in chromosome preparations of seemingly normal persons who later, developed malignancy and also, in $70 \%$ metaphases of recurrently aborting women and in $90 \%$ chromosome preparations of cancer patients. Hence, this is hypothesized that due to hyper activity of some gene combination within the intra-embryonic tissue, a kind of mutational mechanism might be triggered. In incidences of vanishing twin, where the twin pregnancy gradually removes one foetus (finally resulting in a single birth), those induced pre-malignancy features are rejected by selective intrauterine immunogenetic and molecular defence mechanisms. Quite likely, the twin pregnancy resulting in vanishing twin might be a Dizygotic (DZ) pregnancy hence developing embryonal tissue differed in gene-interacting mechanisms.
\end{abstract}

\section{Introduction}

An abortion is not a commonly noticed physiological phenomenon but is a universal incidence of female reproductive outcome. But in our population, there is, may be a small fraction, of such women who abort many a times before successfully maturing and safely delivering the child. Some of them, may not be fortunate to become mothers at all. Termination of pregnancies at an early stage (abortion- before 8-10 weeks or so) or in later maturing months (still births) can be due to many reasons which include external environmental factors (nutritional, physical conditions, drugs etc). Certain nutrients ingested in large quantity, more so vitamins act as drugs but some combinations for example vitamin A derivative accutane causes abortions. Excess consumption of vitamins $\mathrm{A}$ and $\mathrm{C}$ induces many defects in the developing foetus hence pregnant women are often advised for only prescribed doses. On the other hand, malnutrition and vitamin deficient diet further results in abortions [1]. Among internal factors main are anatomical or gynaecological and physiological. When all these parameters are examined and found to be by and large adequate, attention goes to involvement of genetic factors. Such an approach becomes absolutely essential when medical doctors find recurrently aborting women. Recurrent abortions result in to physiological, psychological and sociological trauma not only to the woman but affects the family as a whole. When we consider couple as a unit for investigation this becomes imperative to investigate the family history as well as genetic status of marriages. Inbreeding levels of either parent is important and more important becomes specific marriage which might be consanguineous. Higher inbreeding levels have been related to infertility, foetal wastages and recurrent abortions as well as twinning rates [2-5]. Our inferences on the basis of family studies had opined that recurrent abortions are based on inbreeding status of couples. This inference was based on studies on tribal populations of Central India as well as find of higher abortion rate among families with higher inbreeding coefficient [6]. Subsequent to these studies we also opted for population cytogenetic studies and carried out extensive chromosomal survey studies (during 1984-1998) which included 212 apparently normal individuals, 60 mentally handicapped children and adults, 82 methylisocyanate gas exposed persons (Union carbide tragedy, Bhopal, mid night $2^{\text {nd }}-3^{\text {rd }}$ Dec 1984), 86 persons with syndromes and diseases, and 165 individuals complaining about fertility and abortions. Standard protocols were followed on lymphocyte cultures for chromosome studies using different culture media and staining procedures included simple Giemsa, orcein and Feulgen's techniques; a few slides of each category were also processed for $\mathrm{C}$ and $\mathrm{G}$ banding [7-10]. While scrutinizing and carefully repeating observations on slides we had noticed the presence of marker dots even among the frequently aborting women as well as among cancer

Correspondence to: Kishore Goswami, Bhopal University, MP, India, E-mail: hitkishoreg@gmail.com

Received: September 30, 2017; Accepted: October 27, 2017; Published: October 30,2017 
patients. To investigate further, a proforma to collect information on couples showing repeated abortions was designed and we had finally reported that cancer history of preceding two generations on either maternal or paternal side might be assisting in aborting the installed foetus before 12 weeks of pregnancy [11].

In other words, an obvious hypothesis finds a support that since the foetus has genes from both the parents, an undesired molecular activity supersedes normal timely function of genes resulting in early termination of pregnancy. Chromosome studies from these couples had indicated presence of marker dots [12-14]. Exactly similar situation has been observed in cases of vanishing twin where the twin pregnancy gradually removed one foetus, finally resulting in a single birth. Both parents showed marker dots in addition to a few chromosome aberrations including translocations [15]. Quite likely, these induced pre-malignancy features are rejected by intrauterine immunogenetic and molecular defence mechanisms thereby terminating developing embryonic mass.

\section{Material and methods}

The couples in the age range of 25 to 50 years were referred to the genetic counseling clinic then run at the department of genetics at Bhopal University, Bhopal (MP) for chromosomal screening, during 1984-98 as our laboratory was actively working on multidisciplinary investigations related to blood genetics. Standard protocols were followed on lymphocyte cultures using different culture media and staining procedures included simple Giemsa, orcein and Feulgen's techniques; a few slides of each category (Tables 1 and 2) were also processed for $\mathrm{C}$ and $\mathrm{G}$ banding. These details have been mentioned in earlier publications [7,12,16-19]. Blind scoring of slides continued for a long time in search of chromosomal aberrations with alarming attention for "marker dots"; the chromatin dots discovered and observed since 1973 [9]. A questionnaire was filled up to know the relationship of aborting women with the cancer history if any in the family [11]. Besides many information required the main question was" Has there been any cancer history in the nearest family relative? Pedigrees were constructed to estimate the proportion of common genes to either husband or wife with the cancer patient. This effort gave an information on 281 couples including controls within the sample. Among these 179 couples revealed affiliation with cancer and recurrent abortions. Present specific data to show distribution of couples showing cancer history related abortions among examined persons (Table 1 and 2).

\section{Observations}

Among 281 couples, 179 couples revealed history of cancer in two preceding generations and sometimes in the same generation among first degree relatives of both partners (Table 1). There were 35 couples who had negative history of cancer in previous generations. Also 67 couples out of 281 did not have any abortions and also with negative affiliation with cancer history. Among 179 couples, 31 male partners and 59 female partners had history of cancer in the family; 89 couples had history in the families of both partners Statistically, familial cancer among the female partners is significantly higher $(\mathrm{p}<0.01)$ among male partners. Statistically, data tabulated the value of chi-square $\left(\chi^{2}\right) 6.635$ is very significant and justifies our inference that cancer history in the parentage of either partner (husband or wife) or both does influence abortions (Tables 1 and 2). The commonest information gathered on type of cancer prevalent in the family corresponded to female breast, cervix and lung cancers while the male partners often reported on colon and lung cancers with two on bone cancers. Most of them did not have authentic medical records with them hence this was not further questioned or investigated.
Table 1. Couples studied in relation to abortions and cancer history in the family

\begin{tabular}{|c|c|c|c|}
\hline Cancer History & Abortions & No Abortions & Total \\
\hline $\begin{array}{c}\text { Without cancer } \\
\text { history }\end{array}$ & 35 & 37 & 72 \\
\hline With cancer history & 179 & 30 & 209 \\
\hline Total & 214 & 67 & 281 \\
\hline
\end{tabular}

Table 2. Distribution of 281 couples in relation to cancer history in the family Cancer History

\begin{tabular}{|c|c|c|c|c|}
\hline $\begin{array}{c}\text { Number of } \\
\text { Abortions in } \\
\text { couples }\end{array}$ & With husband & With wife & With both & $\begin{array}{c}\text { Without any } \\
\text { cancer history }\end{array}$ \\
\hline $\begin{array}{c}\text { No abortions } \\
(67)\end{array}$ & 13 & 15 & -- & 37 \\
\hline $\begin{array}{c}\text { 1-2 Abortions } \\
(21)\end{array}$ & 02 & 02 & -- & 17 \\
\hline $\begin{array}{c}3-4 \text { Abortions } \\
(88)\end{array}$ & 18 & 49 & 11 & 10 \\
\hline $\begin{array}{c}5-6 \text { Abortions } \\
(74)\end{array}$ & 10 & 52 & 12 & -- \\
\hline $\begin{array}{c}7 \text { \& more } \\
(31)\end{array}$ & 04 & 11 & 08 & 08 \\
\hline
\end{tabular}

\section{Discussions}

Genes begin to function soon after fertilization. Some genic combinations cause severe problems in the embryonic tissue that developing foetus ceases to grow normally and lethal effects terminate pregnancy before 8 to 10 weeks. Some lethal alleles influence even earlier resulting in spontaneous abortion. About $50 \%$ of spontaneous abortions result from variable chromosome number (extra or missing chromosome). Even both gametes (male and female) contribute normal chromosomal complement, aneuploidy or polyploidy can sometime arise by interfered mitotic divisions within the embryonic tissue, obviously resulting in early termination of pregnancy. However probably when the foetal genotype survives and continues to mature it appears that chromosomal or genic combinations may not have been "lethal-enough" to terminate pregnancy within 10 to 12 weeks. Since chromosomal involvement in early wastage has been known for quite some time $[1,10,11,20,21]$ we had scored on many aberrations on all available metaphases of more than 600 persons during our study. Following specific aberrations were scored: chromosome breaks, somatic translocations, acrocentric associations, marker dots, premature centromeric divisions, tandem duplications, hyperploid cells, hypoploid cells, amitotically dividing nuclei and unusual association of chromosomes or chromatids. These details have been mentioned in earlier publications $[7,8,12,16-18]$ Blind scoring of slides continued for a long time in search of chromosomal aberrations with alarming attention for "marker dots"; the chromatin dots discovered and observed since 1973 [9]. Our suspicion regarding affiliation of abortions with cancer history was strongly linked due to marker dots' association with chromosomal mutagenesis and onset of malignancy. Marker dots (Figure1) appeared in $70 \%$ chromosome preparations (metaphases) of recurrently aborting women and $90 \%$ in chromosome preparations of cancer patients.

Lot many chromosomal and gene-disorders and syndromes are known to have arisen in intrauterine journey and observed at the time of birth (congenital) [21]. Several hundred defective traits develop as an individual grows depending upon the age of onset of genes. So logically, molecular cytogenetic activity, which culminates in to spontaneous abortion quite often in a woman must be a localized mutational activity within the foetus depending upon the resultant embryonic tissue vide genetic contribution of the couple. And such couples who 


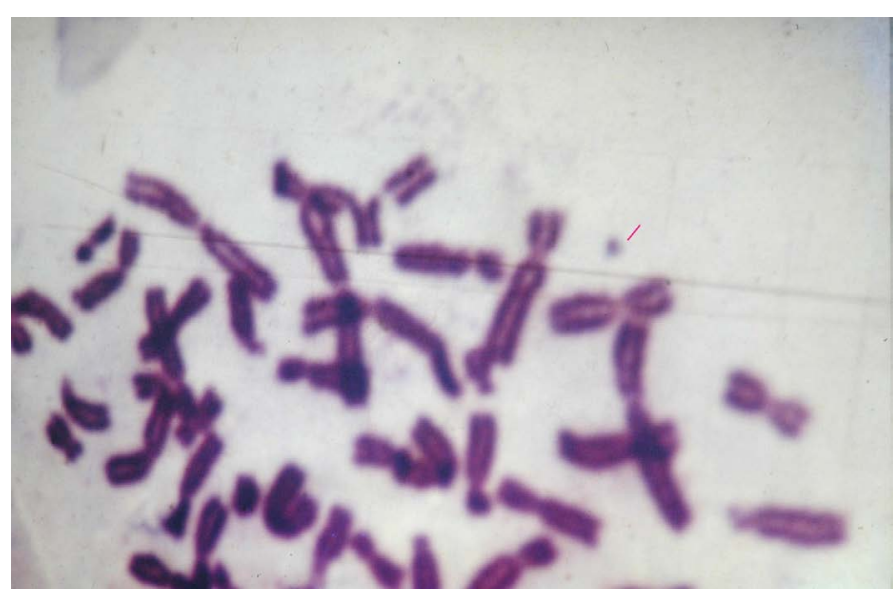

Figure 1. A part of Feulgen-post aceto-orcein stained metaphase from a female patient with cancer history in the family. Note a rare "Levanian translocation "exhibiting two chromatids of a chromosome showing attached to chromatids of two different chromosomes. Marker dot is arrowed. She had reported six abortions in two years. (Photgraphed $15 \times 100$ magnification; enlarged;- After Goswami).

show recurrent abortions more than three times are often possessive of marker dots in their chromosomal complement. Obviously, presence of marker dots in majority of recurrently aborting women must be the cause or consequence of some kind of chromosomal mutagenesis... Our studies on cytogenetics of vanishing twin [15] also have laid greater blame on adverse effects of marker dots. These "marker dots" were observed in seemingly normal persons (both sexes) but later, after a gap of 2 to 6 years, some of them exhibited signs of malignancy. Since, similar situation has been observed in cases of vanishing twin where the twin pregnancy gradually removed one foetus, finally resulting in a single birth. Both parents showed marker dots [15] in addition to a few chromosome aberrations (Figure1) including translocations. We can simply speculate that chromosomal mutagenesis present in somatic cells of the mother might be interfering normal functioning of the gene complex operative within the developing embryo at an early stage. According to a very large International group of multidisciplinary investigators [22] who have made a genome-wide analysis, have recently identified 12 loci influencing human reproductive behavior. Though, both parents contribute genes, it is always the mother's genotype and intrauterine hormonal and nutritional status and other environmental conditions that finally help the developing foetus. So, if everything is optimum, repeated abortions in a woman support an idea that some undesired molecular activity supersedes normal and timely function of genes resulting in early termination of pregnancy. May be, these induced pre-malignancy features are selectively rejected by intrauterine immunogenetic and molecular defence mechanisms thereby terminating developing embryonic mass in mothers reporting vanishing twin and retaining the other embryo. Quite likely, the vanishing twin might be a Dizygotic (DZ) pregnancy to begin with hence differed in gene-interacting mechanisms. So, in incidences of vanishing twin, the induced pre-malignancy features (chromosomal mutagenesis) are rejected by selective intrauterine immunogenetic and molecular defence mechanisms, on interaction with embryos' genetic make-up, thereby terminating one embryonic mass and retaining the other. The other embryo survives selection through immunogenetic defence mechanisms, which by all means, must be deprived of sub lethal combinations.

\section{References}

1. Lewis R (1997) Human Genetics: Concepts and Applications. WCB Mc-Graw-Hill New York, PP 427.

2. Gedda L and Parisi P (1970) The impact of Inbreeding on Human Fertility: considerations on Natural Selection. Proc Sixth World Congress on Fertility and Sterility Tel Aviv 1968. Academy of Sciences \& Humanities Jerusalem

3. Goswami HK (1970) Studies on twins. IV. Twinning in Madhya Pradesh. Acta Genet Med Gemellol (Roma) 19: 465-471. [Crossref]

4. Goswami HK (1987) Twinning and inbreeding in India: the fraternal component. Acta Genet Med Gemellol (Roma) 36: 343-347. [Crossref]

5. Goswami HK (1993) Marker dot is indicator of chromosomal mutagenesis. Bionature 13: 325-333.

6. Goswami HK (1983) Genetics \& Public Health. Some considerations in Indian context. In: Genetics \& Public Health: Catholic Press, Ranchi.

7. Goswami HK (1986) Cytogenetic effects of methyl isocyanate exposure in Bhopal Hum Genet 74: 81-84. [Crossref]

8. Goswami HK (1993) Marker dot is indicator of chromosomal mutagenesis. Bionature 13: $325-333$.

9. Goswami H K (2016) Cells with Chromosomal Aberrations Trigger Neoplastic Transformations in Humans. Twin Research and Human Genetics 19: 77-79.

10. Mandal PK, Rangnekar A, Sengupta L K and Goswami H K (1993) Chromosomal involvements in human Reproductive wastages: An Overview. Bionature 13: 297-317.

11. Rangnekar A, Goswami HK, Goswami R (2004) Recurrent abortions are related to Cancer history. Bombay Medical Journal.

12. Goswami, H. K. (2001). Genetic significance of marker dots. Perspectives in Cytology \& Genetics 10: 265-269.

13. Goswami HK, Rangnekar GV, Varshney S, Gandhi P, Jain B, et al. (1992) Crossed Renal Ectopia with pelvic lipomatosis: A new Syndrome involving Chromosome 1. Human Genetics 89: 666-670

14. Rangnekar A, Goswami HK, Goswami R (2004) Genome-wide analysis identifies 12 loci influencing human reproductive behaviour. Bombay Hospital Journal 46: 381-383.

15. Goswami HK, Rangnekar A, Sikarwar LS (2011) Population Cytogenetics of Abortions and Vanishing Twin. Indian Journal of Fundamental and Applied Life Sciences 1: $36-42$.

16. Goswami HK, Chandorkar MS, Bhattacharya K, Vaidyanath G, Parmar D, et al. (1990) Search for Chromosomal variations among gas exposed persons in Bhopal. Human Genetics 84: 172-176.

17. Goswami HK, Shrivastava N, Gopal SK, Sharma S, Chandorkar MS, et al. (1997) Unusual chromosomal features in a child with gradual disappearance of Right Ulna (mono ostolic osteolysis). J Genet Med 1: 11-16.

18. Goswami HK, Rangnekar AG, Sharma S, Varshney S, Lee IH et al. (1998) Hemihypertrophy with hypomelanosis of Ito: A new syndrome combination. $J$ Genet Med 2: 1-5.

19. Goswami HK, Chang SI (2001) Marker dots are expelled by attenuation in heterochromatin of a chromatid. Bionature 21: 41-48.

20. Mameli M, Cardia S, Milla A, Aste A, Santucci S, et al. (1984) Cytogenetic study in fifty couples with recurrent abortions. Gynaecol Obster Invest 17: 84-88.

21. Vogel F and Motulsky AG (1986) Human Genetics: Problems and Approaches (2nd Edn). Springer Verlag, Berlin pp 807. [Crossref]

22. Barban N, Jansen R, Vlaming R, Vaez A, Mandemakers JJ, et al. (2016) Genome-wide analysis identifies 12 loci influencing human reproductive behavior. Nat Genet 48 : 1462-1472. [Crossref]

Copyright: (C2017 Goswami K. This is an open-access article distributed under the terms of the Creative Commons Attribution License, which permits unrestricted use, distribution, and reproduction in any medium, provided the original author and source are credited. 